\title{
Discrimination of the order of the components of repeating tone sequences: Effects of frequency separation and extensive practice*
}

\author{
R. S. NICKERSON† and B. FREEMAN \\ Bolt Beranek and Newman Inc., 50 Moulton Street, Cambridge, Massachusetts 02138
}

\begin{abstract}
The Ss' task was to identify repeating sequences of pure tones that differed only with respect to the order in which the tones occurred. With tones occurring at a constant rate of $5 /$ sec, performance was better when the tones were widely spaced in frequency than when they were less widely spaced. One $\mathrm{S}$ was able, after considerable practice, to distinguish among different sequences whose component tones were presented at rates up to $500 / \mathrm{sec}$. It was tenatively concluded that, in this case, performance was based on temporal (order) information at the slowest presentation rates, primarily on spectral information at the highest rates, and on both order and spectral information at intermediate rates.
\end{abstract}

It has been known for some time that listeners can discriminate the order of occurrence of two auditory stimuli whose onsets differ by as little as $15-20 \mathrm{msec}$ (Hirsh, 1959). More recently, Patterson and Green (1970) have demonstrated that, under some conditions, ordered pairs can be discriminated even when the interval between onsets is as short as $2 \mathrm{msec}$. In view of the temporal resolving power implied by these results, the recent finding (Warren, Obusek, Farmer, \& Warren, 1969) that, for listeners to be able to report the order of occurrence of the components (usually four) of a repeating auditory sequence, the interval between the onsets of successive components had to be several hundred milliseconds was a surprising one. To be sure, this result was obtained with unpracticed listeners and reflected how well they could do the first time they listened to such a sequence. However, several investigators who have followed up the finding have verified that even with highly practiced listeners the order of the components of a repeating sequence often is not reported accurately if the components are occurring at rates much higher than about $5 / \mathrm{sec}$.

The experiments that have been prompted by Warren's result have produced several findings, among them the following. Speech and speech-like sounds (spoken digits, vowel segments) are easier to order than are nonspeech-like sounds (Thomas, Hill, Carroll, \& Garcia, 1970; Warren, Obusek, Farmer, \& Warren, 1969). Sequences of contiguous pure tones

\footnotetext{
*This work was supported by the Air Force Office of Scientific Research under Contract No. F44620-69-C-0115. The authors are grateful to David Green, Barry Leshowitz, Kenneth Stevens, and Richard Warren for helpful comments on the manuscript.

†Address reprint requests to R. S. Nickerson, 50 Moulton Street, Cambridge, Massachusetts 02138.
}

are easier to order when each tone glides smoothly into the next than when the transition between successive tones is abrupt [Dannenbring, 1971 (reported in Wilcox, 1971)]. For a given rate of occurrence of sequence components, performance is better when silent intervals are inserted either between successive components, or between cycles of the sequence, or both (Neisser, 1972; Thomas, Cetti, \& Chase, 1971; Warren, 1972a). The ease with which tone sequences can be ordered depends in part on the frequency separations among the tones of the sequence (Bregman \& Campbell, 1971; Thomas \& Fitzgibbons, 1971). For a given set of components, some orderings may be more easy to discriminate than others (Neisser, 1972; Warren \& Obusek, 1972).

One of the problems associated with this area of research is illustrated by the fact that the accuracy with which one can report order depends in part on the method of reporting that is used. Warren and Obusek (1972) found, for example, that listeners could do better if they were required to indicate the order of the sequence components by arranging cards on a table than if they were required to specify their order verbally, although even with the more effective method of report, performance was at a chance level when components occurred at a $5 / \mathrm{sec}$ rate. One plausible explanation of this effect is that the card-arranging method of report imposes less of a load on memory and consequently makes it possible for the listener to adopt a different and more effective listening strategy than can be used when the report has to be verbal. The method permits the listener to attend to the components of a sequence a pair at a time, to represent the order of the components of a pair with the corresponding cards, and then to shift attention to another pair, possibly containing one of 
the components of an already-ordered pair, and so on. In principle, he could adopt the same listening strategy with the verbal report; in this case, however, he would have to remember the pairwise relationships that had already been determined, a task that could interfere with listening.

The present study was motivated in part by the conjecture that one may be able to discriminate order even when he cannot accurately report it, and that the reason for this limitation is that the listener is unable to translate the perceived sequence into the required output code. This notion is closely related to Neisser's (1972) distinction between "strings" and "analogs," two forms in which he assumes that a sequence may be represented in the listener's memory. According to this conceptualization, when a sequence is presented slowly enough, one can represent it as a "string" of verbal signs of the components that is isomorphic to the components themselves with respect to their order. When a sequence occurs too rapidly for the listener to encode it as a string in real time, he represents it as an "analog" which consists of a set of distinctive features of the sequence. One can use an analog representation as the basis for comparing one sequence with another that is subsequently presented, but one cannot report the order of the components of an analog representation directly. To report order, one must construct a string representation on the basis of the cues that he can derive from an analysis of the analog. Warren (1972b) makes a similar distinction. He suggests that the lower limit (in terms of duration of sequence components) for the direct identification of temporal order is determined by the maximum rate at which one can attach verbal labels to the successive components as they occur in real time, and he places this limit somewhere in the vicinity of $200 \mathrm{msec}$ per component. Thus, if one is to identify the order of components when they occur at a rate greater than about $5 / \mathrm{sec}$, Warren would say that he must do so via a two-stage process. He must first recognize a larger temporal pattern that incorporates the components, and then infer the order of the components on the basis of a previously learned rule describing their internal arrangement.

A basic question that is suggested by these distinctions is whether the maximum rate at which one can verbalize imposes a limitation only on one's ability to report order, or on his ability to discriminate it as well. One procedure that has been used to study order discrimination that is not encumbered by response-encoding considerations is the "same""different" judgment. In this case, two sequences are presented, either successively or alternately, and the listener attempts to decide whether the components occur in the same order in both sequences. Performance is very much better than when the order of occurrence must be reported directly (Wilcox, 1972; Wilcox, Neisser, \& Roberts, 1972). Warren (1972b), for example, has shown that highly trained listeners are able to do very well on a "same". "different" task with sequences comprising contiguous components with durations as short as $5 / \mathrm{msec}$. As a method for investigating discrimination limitations independently of output-encoding limitations, however, this procedure has a major drawback, because it is not necessary to discriminate the order of two sequences to determine that they differ. A detection of a difference in the ordering of any two components suffices to indicate that the order of the sequences is not the same. One might argue that this is not true in the case of determining that the components of the two sequences are ordered alike. However, inasmuch as it is customary to have "same" and "different" pairs occur equally frequently in an experimental run, the fact that one of the decisions is relatively easy to make may well have an effect on the accuracy with which the other decision is made also.

An attempt was made to circumvent outputencoding limitations in the present experiment by using an absolute judgment paradigm. Listeners were trained to identify each sequence by labeling it rather than by reporting the order of its components. In this case, it is not possible to discriminate among all the sequences accurately by attending to a limited set of pairwise relationships.

Another motivation for the experiment was the question of the effect of the frequency separation among component tones on the discrimination of their order. As noted, this variable has been studied by Thomas and Fitzgibbons (1971) and by Bregman and Campbell (1971). Thomas and Fitzgibbons found that order could be determined more easily if the tones were within a musical fourth than if they were more widely spaced. Performance in the former case was similar to that obtained with sequences of natrral vowels, whereas performance in the latter case was more nearly like that obtained with sequences of qualitatively different sounds.

Bregman and Campbell investigated a phenomenon that they described as sensory-stream segregation. Using sequences of six $100-\mathrm{msec}$ tones, three of relatively high frequencies $(2,500,2,000$, and $1,600 \mathrm{~Hz})$ and three of relatively low frequencies $(550$, 430 , and $350 \mathrm{~Hz}$ ), they obtained evidence that the tones were perceived as two separate streams, one comprising the high frequencies and one the low. Order discrimination was better within than across streams. Bregman and Campbell's explanation of the relative difficulty of discriminating order across streams is similar to that proposed by Thomas and Fitzgibbons to account for the relative difficulty of sequences composed of qualitatively different sounds. In the one case, performance is assumed to be limited by the time-consuming process of shifting attention from stream to stream; in the other, the need to shift attention from one stimulus class to another is what is 
assumed to slow things down.

The results of Thomas and Fitzgibbons, and of Bregman and Campbell, would lead one to expect that the difficulty that one should have in discriminating the order of pure tones should be some monotonically increasing function of the magnitude of the frequency differences among the tones (assuming, of course, that the smallest frequency differences that are used are large enough so that the tones are readily distinguishable). Moreover, the attention-switching hypothesis would also lead to that expectation, if one assumes that the time required to switch attention from one tone to another is a monotonic function of the difference between their frequencies-an assumption that has been found to be a useful one in other contexts.

\section{METHOD}

Stimuli were repeating sequences of four tones. Each sequence cycled for $4 \mathrm{sec}$. The six possible orderings of four tones in a repeating sequence are shown in Fig. 1 . The starting points are arbitrary in this figure; in the experiment, the starting tone for a given sequence was selected randomly on each trial. ${ }^{1}$

The lines in Fig. 1 are an expository convenience and are not intended to represent a musical seale. Throughout the remainder of this report, the numbers 1 through 6 will be used to designate the specific orderings with which they are associated in the figure. The letters A, B, C, and D are used to represent the individual tones of a sequence, from lowest to highest, respectively.

Three frequency sets were used to generate the stimulus sequences. The first, or medium, set (M) comprised four tones spaced at approximately one-third octave: $469,586,732$, and $916 \mathrm{~Hz}$. The second, or wide, set (W) contained both lower and higher frequencies than the first; the two lower tones, 300 and $375 \mathrm{~Hz}$, differed by one-third octave, as did the two higher tones, 1,144 and $1,431 \mathrm{~Hz}$, whereas the separation between the two middle tones was one and two-thirds octave. The tones of the third, or narrow, set $(\mathrm{N})$ were all contained within a one-third-octave range: $586,631,680$, and $732 \mathrm{~Hz}$.

Each of six Ss (paid high school students) had 25 test runs with each frequency set. The order in which $\mathrm{Ss}$ encountered the three frequency sets was counterbalanced.

Each $S$ participated in the experiment on 2 successive days. On Day 1 , he had for each frequency set 1 "listening" run followed by 10 "test" runs. The purpose of the listening run was to familiarize $S$ with the six repeating sequences that could be generated with a given set of tones. He was told to associate a different number (1 through 6) with each sequence. The sequences were presented four times each in the order $1,2, \ldots 6,1,2, \ldots$ Immediately following the listening run, the 10 test runs were presented. Each test run was composed of 6 listening trials, in which the six sequences were presented in the order 1 through 6 , and then 18 test trials, in which each of the six sequences occurred three times in random order. On Day 2, one listening run was followed by 15 test runs. The data that are reported were obtained on the test trials of the 25 test runs.

On the test trials, $S$ indicated his choice by typing the appropriate number on a computer terminal. He was allowed to take as much time as he wished, following the termination of the stimulus sequence, before making his response; and he could change his response if he wanted to do so. When satisfied with his choice, he typed a comma, and the number immediately preceding the comma was taken to be his response. Feedback (the correct number) was given immediately following each response. A PDP-8/E computer was used to generate the stimuli, collect the responses, and present the feedback.
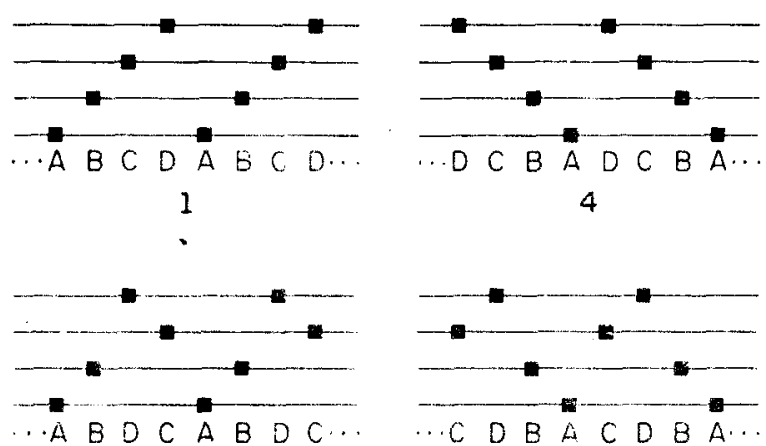

2
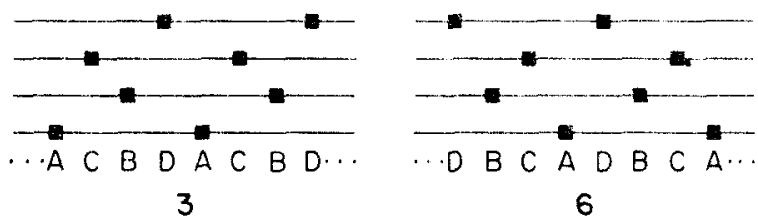

Fig. 1. The six possible orderings of four tones. The starting places that are shown are arbitrary and were chosen to show the symmetry of Sequences 1 and 4,2 and 5 , and 3 and 6 . In the experiment, the starting tone for a given sequence varied randomly from trial to trial.

Stinuli were adjusted to a comfortable listening level and delivered binaurally over earphones. Because the digital method that was used to generate the tones produced high-frequency artifacts, the sequences were passed through a low-pass filter with a cutoff at $1.600 \mathrm{~Hz}$.

\section{RESULTS}

The 25 test runs for each $S$ were grouped in five blocks of 5 successive runs each. An analysis of variance was performed using the total number of correct identifications by an individual $S$ during a 5 -run block as the basic performance measure. All main effects were significant: the frequency spread among the tones from which the sequence was composed, $F(2,10)=4.36, p<.05 ;$ the order of tones. within a sequence, $F(5,25)=3.34, \mathrm{p}<.025$; and practice, $F(4,20)=19.58, p<.01$.

The effects of frequency spread and of practice are shown in Fig. 2. The fact that performance was better for sequences composed of tones of widely different frequencies than for those composed of tones within a narrower range is surprising in view of the results obtained by Thomas and Fitzgibbons (1971) and by Bregman and Campbell (1971). The lack of a significant interaction between frequency spread and practice supports the conclusion that this difference in the results of those experiments and the present one is probably not due to Ss' performing at different levels of skill.

The effect of tone order indicates that the six possible orders were not equally discriminable, and a 
significant Frequency by Order interaction, $F(10,50)$ $=3.89, \mathrm{p}<.01$, suggests that the relative difficulty of the different orderings was not the same for all frequency sets. The possibility that the main effect of tone order is attributable in part to response biases is ruled out by the fact that the six possible responses occurred with nearly equal frequency, the average (absolute) deviation from the expected $16.7 \%$ being less than $1 \%$. The individual differences among Ss preclude a definitive ordering of the tone orders in terms of difficulty; however, it does appear that for most Ss the two "continuous" orders (ABCDAB ... and $\mathrm{DCBADC} . .$.$) were somewhat more readily$ discriminated than were the other patterns.

It was apparent from an inspection of the confusion matrices that the confusions that were made were not random. In particular, Sequences 3 and 6 (ACBD and DBCA) formed a class apart from the others. These sequences were confused very infrequently with any of the remaining four: the probability that the response would be 3 or 6 , given that the stimulus was 3 or 6 , was .88 ; the probability that the response would be either 3 or 6 , given that the stimulus was $1,2,4$, or 5 , was about .06. However, 3 and 6 were hardly distinguishable from each other. Letting $p(R \mid S)$ represent the probability that the response would be $\mathrm{R}$, given that the stimulus was $\mathrm{S}, \mathrm{p}(3 \mid 3)=.50$, $\mathrm{p}(6 \mid 3)=.38, \mathrm{p}(6 \mid 6)=.43, \mathrm{p}(3 \mid)=.44$. No two other sequences came close to being as mutually confusable as these two. For the nearest contenders, the probability of a confusion was .17 .

\section{SOME SUPPLEMENTARY DATA}

Two subsequent experiments were performed for the purpose of exploring effects of covarying the durations of the tones and the durations of the silent intervals between successive tones. Only set (M) was used, and the presentation rate was again constant at 5 tones/sec. The results were inconclusive; however, in both cases, the interstimulus confusions that were obtained were very similar to those described above. In particular, Sequences 3 and 6 were highly distinguishable from the other sequences but were nearly indistinguishable from each other. The salient data are shown in Table 1. Data from the main experiment are included for comparison.

\section{DISCUSSION}

The results bear out the general finding that listeners are not very good at distinguishing the order of tones that are delivered at the rate of about $5 / \mathrm{sec}$, even when the response procedure is such as to minimize the output-encoding demands. None of the listeners that were used in this experiment was given really extensive practice with the task, however, and we will return to the question of the effect of

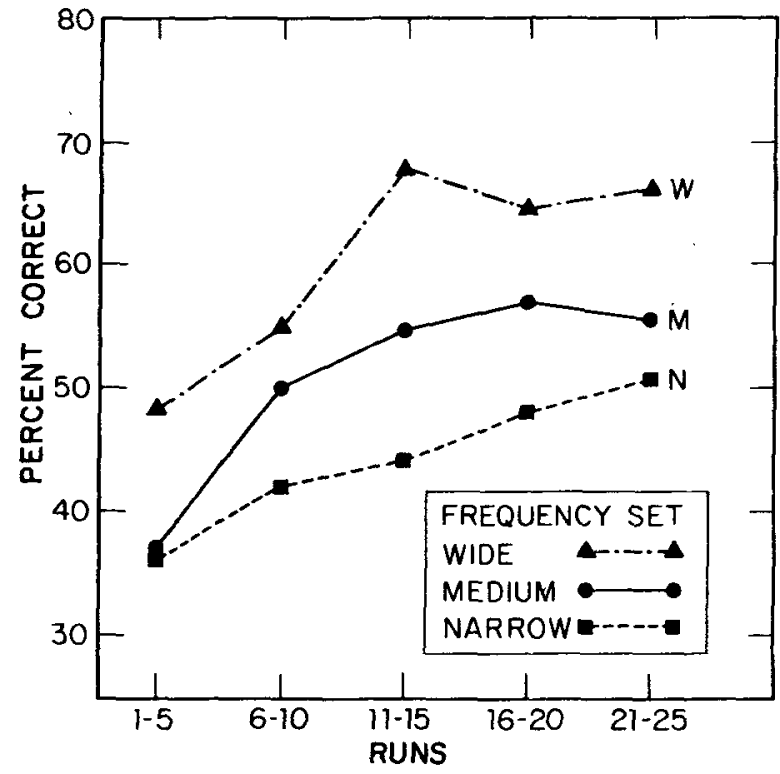

Fig. 2. Percept correct identifications as functions of practice for each of the three frequency sets.

prolonged practice presently. Here we will focus on the implications of the way in which performance varied with the experimental conditions rather than on the absolute levels obtained. In particular, we need to account for the effect of frequency separation and for the types of intersequence confusions that occurred.

Our explanation for both of these effects invokes the notion of selective listening and assumes that the listener has some flexibility with respect to the listening strategy that he adopts. Suppose that what the listener does is to attend to different aspects of a sequence at different times. In particular, suppose, as is suggested by Bregman and Campbell's (1971) stream-segregation result, that he listenes at any given time to either the two higher frequency tones or to the two lower frequency tones, but does not attend to both pairs simultaneously. Suppose, further, that he is able

Table 1

Percentages of Specific Types of Confusions and Correct Responses Obtained in the Main and Two Subsequent Experiments

\begin{tabular}{lcll}
\hline \multicolumn{1}{c}{$\mathrm{p}(\mathrm{R} I S)^{*}$} & $\begin{array}{c}\text { Main } \\
\text { Experiment }\end{array}$ & A & Bubsequent \\
\hline $\mathrm{p}(3$ or $6 \mid 3$ or 6$)$ & .88 & .89 & .76 \\
$\mathrm{p}(3$ or $6 \mid 1,2,4$, or 5$)$ & .06 & .06 & .13 \\
$\mathrm{p}(3 \mid 3)$ & .50 & .48 & .41 \\
$\mathrm{p}(6 \mid 3)$ & .38 & .40 & .34 \\
$\mathrm{p}(6 \mid 6)$ & .43 & .60 & .39 \\
$\mathrm{p}(3 \mid 6)$ & .44 & .32 & .39 \\
\hline
\end{tabular}

Note-In Experiment $A$, each of four $S_{s}$ had 25 test runs; in Experiment $B$, each of five Ss had 10 test runs.

*Probability of specified response given specified stimulus. 
to discriminate differences in spacing between the two tones to which he attends, can therefore distinguish, for example among $\mathrm{AB}--\mathrm{AB}--$, $\mathrm{A}-\mathrm{B}-\mathrm{A}-\mathrm{B}$, and $\mathrm{BA}-\mathrm{BA}-\mathrm{-}$. Such a listening strategy should produce several effects.

First, note that Sequences 3 and 6 can be readily distinguished from the other four sequences on the basis of comparison of either the high-frequency or the low-frequency tones (see Fig. 1). For both 3 and 6, the two high-frequency tones alternate at equally spaced intervals, and this is not true of any other sequence. The same observation may be made with respect to the two low-frequency tones. Note also, however, that Sequences 3 and 6 are identical with respect to the temporal pattern of their high-frequency tones and also with respect to that of their low-frequency tones. It is only by relating the high-frequency and low-frequency tones with each other than one can distinguish between these two sequences. Thus, these two sequences should be easily distinguished from the other four sequences, but highly confusable with each other.

Consider now the four remaining sequences. Two pairs (1 and 5,2 and 4) are identical with respect to their high-frequency tones and differ with respect to their low-frequency tones, whereas two other pairs (1 and 2,4 and 5) are the same with respect to their low-frequency tones but differ with respect to their highs. Thus, if one listens only to the two higher frequency tones, one will confuse Sequences 1 and 5 , or 2 and 4 ; whereas, if one listens only to the two lower frequency tones, the sequences that will be confused are 1 and 2 , or 4 and 5 . In order to identify one of these sequences uniquely, the listener must attend both to the high tones and to the low. It is not necessary that he relate the one set to the other, however, In principle, he should be able to narrow his range of alternatives to two by listening first to one of these pairs, and then to eliminate one of these two possibilities by listening to the other pair.

Two pairs of sequences ( 1 and 4,2 and 5) differ with respect to both low- and high-frequency tones. Thus, because it is not necessary to listen to both the high- and the low-frequency pairs to discriminate between Sequences 1 and 4 , or between 2 and 5 , one might expect the discrimination between the members of both of the latter two pairs to be somewhat better than between those of any of the other four possible pairings of these sequences.

Finally, if the hypothesized listening strategy is adopted, and if it is effective at all, one would expect its degree of effectiveness to be greater, the greater the frequency separation between the high- and low-frequency groups.

According to this line of reasoning, the 15 possible confusions that can occur (we ignore the directionality of the confusion, so responding " 3 " when the stimulus is 4 and responding " 4 " when it is 3 are both
Table 2

Number of Confusions Between Specified Pairs: Main Experiment

\begin{tabular}{lccc}
\hline & \multicolumn{3}{c}{ Range of Frequencies in Sequence } \\
\cline { 2 - 4 } & Narrow & Medium & Wide \\
\hline Unlikely & & & \\
$3-1$ & 51 & 20 & 9 \\
$3-2$ & 74 & 24 & 8 \\
$3-4$ & 44 & 19 & 9 \\
$3-5$ & 64 & 30 & 10 \\
$6-1$ & 33 & 19 & 10 \\
$6-2$ & 47 & 22 & 5 \\
$6-4$ & 51 & 25 & 6 \\
$6-5$ & 62 & 28 & 4 \\
$1-4$ & 60 & 55 & 59 \\
$2-5$ & 193 & 135 & 97 \\
Mean & 67.9 & 37.7 & 21.7 \\
Mean without & 53.3 & 23.4 & 7.6 \\
$1-4$ and 2-5 & & & \\
Moderately Likely & & 92 & 86 \\
$1-2$ & 134 & 132 & 110 \\
$1-5$ & 145 & 202 & 116 \\
$2-4$ & 120 & 144 & 118 \\
$4-5$ & 132 & 142.5 & 107.5 \\
Mean & 132.8 & 384 & 426 \\
Highly Likely & & & \\
$3-6$ & 298 & & \\
\hline
\end{tabular}

considered instances of a confusion between 3 and 4) can be grouped into three categories reflecting their likelihood of occurrence if the hypothesized listening strategy is used. This grouping is shown in Table 2 . Two things are clear from an inspection of this table. First, the pattern of confusions that was obtained is generally consistent with the predicted likelihoods. Second, the effect of increasing the range of frequencies represented in the sequence was to make the "likely" confusions more likely and the "unlikely" ones less likely; that is to say, it made the easy discriminations easier and the difficult ones more difficult still. We conclude, therefore, that selective listening probably did play a significant role in determining performance in this experiment.

Inspection of Table 2 also suggests, however, that the pair of sequences 2 and 5, and possibly also 1 and 4 , may be misclassified with respect to difficulty. The 2-5 pair, at least, appears to be considerably more confusable than pairs having 3 or 6 as one member and $1,2,4$, or 5 as the other. A possible clue to this anomaly may be found in a property that Pairs 1 and 4,2 and 5 , and 3 and 6 share. In each of these cases, the order of the components of one member of the pair is the reverse of that of the other. It is possible that this relationship may be a factor in determining the confusability of sequences that have it, although why this should be so is not clear.

\section{THE EFFECT OF EXTENDED PRACTICE}

The Ss in this study were given a moderate amount of practice, but no attempt was made to see how well 
they might do after extended training. In an effort to determine whether one can learn to discriminate ordered sequences in which the components are presented at much higher rates than $5 / \mathrm{sec}$, a single $S$ (the second author) was given several hundred runs over a period of several months.

The $\mathrm{S}$, who had had considerable experience listening to sequences of the general type used, ran herself on an ad lib schedule as time permitted. The order in which the stimulus sequences occurred was randomized by the computer for each run, so, although $S$ was not "naive," her familiarity with the experimental procedure did not permit her to predict the trial-by-trial stimuli.

A given rate of stimulus presentation was used until $S$ managed at least two successive runs with an error rate of not more than $10 \%$. The rate of presentation was then increased and she began again. Irrespective of the rate of presentation, the sequence was repeated for $4 \mathrm{sec}$ on each trial.

The frequencies of the tones were those of the $M$ set. The durations of all components were equal, and a silent interval of the same duration separated successive tones. All sessions were test sessions and were composed of 6 listening trials followed by 30 test trials.

Figure 3 shows percent-correct sequence identifications as a function of number of runs for each component presentation rate. All but two of the curves are taken to a criterion of two successive runs for which the percent correct was 90 or more. The curves representing presentation rates (tones/sec) of 125 and 500 are truncated inasmuch as criterion was reached in these cases in 42 and 49 runs, respectively. $^{2}$

These results supplement Warren's (1972) finding that Ss can do well judging whether two tone sequences are the same or different when the components are presented at rates as high as $200 / \mathrm{sec}$. We have already noted that to determine that two sequences differ, one need only determine that they differ with respect to the order of any two components. Given the response procedure used in the present experiment, one cannot identify the sequences accurately if he restricts attention in this way. Thus, the present results are even more compelling evidence than that provided by "same""different" judgments that differently ordered repeating sequences can be discriminated when the components are presented at rates in the range of a few hundred per second.

From S's comments, it is fairly clear that the basis of the discrimination differs for the slower and the faster presentation rates. At the slower rates, one is aware that the components are occurring in sequence and can focus attention on individual components fairly easily. The individual components of a sequence are not perceptually distinct with the higher rates of

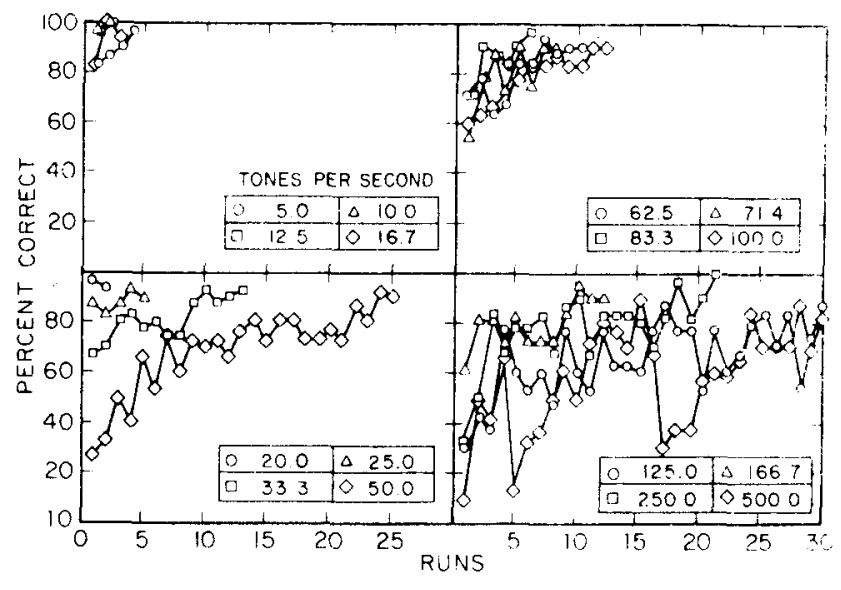

Fig. 3. Percent correct identifications as functions of practice for presentation rates ranging from $5 /$ sec to $500 /$ sec. The $S$ practiced with a given presentation rate until she obtained $90 \%$ correct responses on two successive runs. The criterion was reached in $\mathbf{4 2}$ and 49 runs with presentation rates of 125 and $500 /$ sec.

presentation, however; rather, one hears a steady state sound that has the quality of a tonal buzz.

It seems likely that performance is based primarily on order discrimination for the slower presentation rates and mainly on the detection of spectral differences for the faster rates. The data give only a clue, however, concerning at what rates the transition from one basis to the other takes place. It appears from Fig. 3 that the task was quite easy with the slowest presentation rates and became more difficult as the rate was increased to about 50 components per second. It appears to have become somewhat easier again as the rate was increased to about 62 components per second and somewhat beyond, and then finally to have increased in difficulty again as the rate was further increased to hundreds of components per second.

Our tentative explanation of this pattern is as follows. For the slowest presentation rates used, the discrimination is based largely on order information, and the slower the rate, the better the performance. However, order information becomes less usable as the presentation rate is increased. For the highest presentation rates, the discrimination is based primarily on steady state spectral information. For intermediate rates, we suspect that the listener may be able either to listen for qualitative (spectral) properties or to attend to ordinal relationships among subsets of the tones. Further, we suspect that in these cases the judgment is sometimes based on one type of information, sometimes on the other, and perhaps sometimes on a combination of both.

\section{REFERENCES}

Bregman, A. S., \& Camprell, J. Primary auditory stream segregation and perception of order in rapid sequences of tones. Journal of Experimental Psychology, 1971, 89, 244-249. 
Dannenbring, G. The effect of continuity on auditory stream segregation. Unpublished Master's thesis, McGill University, July 1971 .

Hirsh, I. J. Auditory perception of temporal order. Journal of the Acoustical Society of America. 1959, 31, 759-767.

Neisser, U. On the perception of auditory sequences. Paper given in a symposium, Perception of Temporal Order in Hearing: Old Pattern-Recognition Problems in a New Guise, American Psychological Association meeting, Honolulu, September 1972.

Patterson, J. H., \& Green, D. M. Discrimination of transient signals having identical energy spectra. Journal of the Acoustical Society of America, 1970, 48, 894-905.

Thomas. I. B., \& Fitzgibions, P. J. Temporal order and pereeptual classes. Journal of the Acoustical Society of America, 1971, 50, 86-87. (Abstract)

Thomas, I. B.. Cetti, R. P., \& Chase, P. W. Effect of silent intervals on the perception of temporal order for vowels. Journal of the Acoustical Society of America, 1971, 49, 85. (Abstract)

Thomas, I. B., Hill, P. B., Carroll, F. S., \& Garcia, B. Temporal order in the perception of vowels. Journal of the Acoustical Socicty of America, 1970, 48, 1010-1013.

WARren, R. M. Perception of temporal order: Special rules for the initial and terminal sounds of sequences. Journal of the Acoustical Society of America, 1972a. (Abstract)

Warren, R. M. Temporal resolution of auditory events. Paper given in a symposium, Perception of Temporal Order in Hearing: Old Pattern-Recognition Problems in a New Guise, American Psychological Association meeting, Honolulu, September 1972b.

W ARren, R. W.. \& OBUSEK, C. J. Identification of temporal order within auditory sequences. Perception \& Psychophysics, 1972 , 12, 86-90.

Warren. R. M., Obusek, C. J., Farmer, R. M., \& Warren, R. P. Auditory sequence: Confusion of patterns other than speech or music. Scicnec, 1969, 164, 586-587.

Wilcox, G. W. Temporal coherence of tone sequences. Paper given in a symposium. Perception of Temporal Order in Hearing: Old Pattern-Recognition Problems in a New Guise, American Psychological Association meeting, Honolulu, September 1972.

Wilcox, G., Neisser, U., \& Roberts, J. Recognition of auditory temporal order. Paper presented at the Eastern Psychological Association meeting. Boston, April 1972.

\section{NOTES}

1. Although four tones can be ordered in 24 ways, only six orders are distinguishable, given that the sequence is repeating and its starting point is irrelevant. For example, for our purposes the orders $\mathrm{ABCDAB} \ldots \ldots$ and $\mathrm{BCDABC} . . .$. are the same.

2. Because it was not anticipated that $S$ would learn to identify sequences at such rapid rates, the tone-generating algorithm was written so that each component of the sequence would have an integral number of cycles. A timing counter was consulted by the computer program after the completion of each cycle to see if the desired component duration had elapsed. Thus, the duration of a component could exceed the specified duration by as much as about $2 \mathrm{msec}$ (in the case of the $469-\mathrm{Hz}$ tone), although, on the average. the deviation would be much less than that. This difference was compensated by a shortening of the following intertone interval. With all but the fastest presentation rates, this error was negligible and assumed that all tones started and stopped at the same point in the amplitude-time function (at the down-going zero crossing). With the fastest presentation rates, however, the error could be a significant fraction of the component, or interval, duration. Thus, at the fastest presentation rates the intertone intervals were relatively short and the tones themselves relatively long.

In order to check whether this aspect of the most rapid sequences contributed to their discriminability, the tone-generating algorithm was changed to produce a fixed-time segment of each tone rather than an integral number of cycles. In this case, less than a full cycle of the tones was presented at the fastest presentation rates. However, exactly the same segment of the cycle occurred on every presentation of a given component. With the modified algorithm. S required 33 runs to reach the criterion of $90 \%$ correct with a presentation rate of $500 / \mathrm{sec}$; with the original algorithm. she required 49 runs to reach the same criterion with the same rate. More importantly, she reached the criterion in both cases.

(Received for publication May 7, 1974; revision accepted July 9 , 1974.) 